\title{
SPATIAL LINEAR STABILITY ANALYSIS OF OPEN-CHANNEL FLOWS WITH TRANSVERSE SHEAR REVISITED
}

\author{
Adriano Coutinho de LIMA $^{1}$ and Norihiro IZUMI ${ }^{2}$ \\ ${ }^{1}$ Member of JSCE, Doctor of Engineering, Assistant Professor, Division of Field Engineering for Environment, Fac- \\ ulty of Engineering, Hokkaido University (Kita 13, Nishi 8, Kita-ku, Sapporo, Hokkaido, 060-8628, Japan) \\ ${ }^{2}$ Member of JSCE, Ph.D., Professor, Division of Field Engineering for Environment, Faculty of Engineering, Hok- \\ kaido University (Same address as above)
}

\begin{abstract}
Based upon the shallow water equations, linear stability analysis is conducted on an open-channel flow with velocity retardation on one side. The analysis is applied to a steady turbulent base flow which is characterized by a sub-depth scale turbulence derived from a logarithmic profile of vertical velocity. The linear stability analysis is performed for the case of spatially growing disturbances and compared to the case of temporally growing disturbances. We found that discrepancies between results from the spatial and the temporal approaches were less relevant when the bed friction effect and the kinematic eddy viscosity effect were more significant.
\end{abstract}

Key Words: Spatial linear stability analysis, viscous shear flow, open-channel flow

\section{INTRODUCTION}

Mixing in channels and rivers, which involves transport of momentum and mass and in certain cases heat, is a topic of high current interest, as it is relevant to water quality control, transport of nutrients, sediment and other solutes, erosion processes and biology of aquatic systems. ${ }^{1,2,3,4}$ An accurate prediction of the conveyance capacity and the velocity and boundary shear distributions in channels and rivers is required for practical engineering problems such as flood reduction measures, flood plain management, river restoration and control of sediment and pollutant transport in rivers. ${ }^{5,6}$

In the present work, we focus on the case of a mixing layer excited by a velocity inflection in an open-channel flow. Such transverse shear flows are observed for example in partially vegetated channels, in composite channels, in inflows at channel junctions or in channels where the bottom roughness varies laterally. ${ }^{4,5)}$ The transverse shear results in Kelvin-Helmholtz instability with large-scale horizontal vortices centred around the velocity inflection point. $^{7,8,9)}$

Stability characteristics of transverse shear lay- ers have been studied extensively by means of linear stability analysis. In the classical works of Michalke, ${ }^{10,11)}$ the linear stability analysis was employed for timely-growing and spatially-growing instabilities in an inviscid shear layer. According to Michalke's ${ }^{10,11)}$ results, features observed in the experiments of Freymuth, ${ }^{16)}$ such as a sharp phase reversal of the disturbance velocity and symmetric and anti-symmetric cross-distributions of the real and imaginary parts of the eigenfunction, respectively, could be captured only by the spatial analysis.

Succeeding Michalke, ${ }^{10,11)}$ stability analyses of shear layers with the consideration of effects of bed friction, gravity and kinematic eddy viscosity have been performed, ${ }^{6,7,8,9,12,13,14,15)}$ mostly for the timely-growing case. In the present study, the flow configuration of Lima and Izumi ${ }^{15,17)}$ is modified to consider spatially growing disturbances. The steady turbulent flow formulation of Lima and Izumi, ${ }^{15,17)}$ which has been validated by a comparison with experiments of Refs. 2,9,18, assumes a kinematic viscosity which represents turbulence with a length scale smaller than the flow depth. By doing so, the base flow is set as the flow completely unaffected 
by the shear layer instabilities.

We compare results from the spatial analysis with those from the temporal analysis. The differences among both approaches for viscous flow are found to be less relevant than those for inviscid flow.

\section{FORMULATION}

We focus on horizontal vortices generated in the shallow flow in a wide rectangular open-channel with an inflection point in the depth averaged streamwise velocity. The fluid motion is governed by the following momentum and continuity equations:

$\frac{\partial \widetilde{\boldsymbol{U}}}{\partial \tilde{t}}+(\widetilde{\boldsymbol{U}} \cdot \widetilde{\boldsymbol{\nabla}}) \widetilde{\boldsymbol{U}}=\widetilde{\boldsymbol{g}}-\tilde{g} \widetilde{\boldsymbol{\nabla}} \widetilde{H}-\frac{\widetilde{\boldsymbol{T}}_{b}}{\tilde{\rho} \widetilde{H}}+\tilde{v}_{t} \widetilde{\nabla} \widetilde{\boldsymbol{U}}$,

$\frac{\partial \widetilde{H}}{\partial \tilde{t}}+\widetilde{\boldsymbol{\nabla}}(\widetilde{H} \widetilde{\boldsymbol{U}})=0$,

where $\tilde{t}$ is time, $\widetilde{\boldsymbol{U}}=(\widetilde{U}, \tilde{V})$ is the velocity vector with components $\widetilde{U}$ and $\tilde{V}$ in the streamwise $(\tilde{x})$ and transverse $(\tilde{y})$ directions, respectively, $\widetilde{H}$ is the flow depth, $\widetilde{\boldsymbol{g}}=(\widetilde{g} S, 0)$, where $\widetilde{g}$ is the gravity acceleration and $S$ is the streamwise bed slope, $\widetilde{\boldsymbol{T}}_{b}=\left(\widetilde{T}_{b x}, \widetilde{T}_{b y}\right)$ is the bed shear stress vector, $\tilde{\rho}$ is the water density, $\tilde{v}_{t}$ is the kinematic eddy viscosity and $\widetilde{\boldsymbol{\nabla}}=(\partial / \partial \tilde{x}, \partial / \partial \tilde{y})$. The velocity inflection point is located at $\tilde{y}=0$. The range $-\tilde{B}_{s} \leq \tilde{y} \leq 0$ is denoted as the slow flow zone, and the range $0 \leq \tilde{y} \leq \widetilde{B}_{f}$ is denoted as the fast flow zone, with $\tilde{B}_{s}$ and $\tilde{B}_{f}$ being the widths of the slow and fast zones, respectively. The tilde indicates dimensional variables.

Far from the shear layer, in the fast flow zone, $\tilde{v}_{T}$ should correspond to the sub-depth scale turbulence generated by the bottom friction. We employ the logarithmic velocity distribution as a sub-depth scale turbulent velocity distribution due to the bottom friction. The kinematic eddy viscosity then takes a parabolic form which is depth-averaged, yielding $^{17}$

$$
\tilde{v}_{T}=\frac{1}{6} \kappa C_{f}^{1 / 2} \widetilde{U}_{f} \widetilde{H}_{f},
$$

where $\kappa$ is the Kármán constant $(=0.4), C_{f}$ is the bottom friction coefficient in the fast flow zone and $\widetilde{U}_{f}$ and $\widetilde{H}_{f}$ are the flow velocity and depth, respectively, sufficiently far from the shear layer in the fast flow zone. Although (3) is derived for the zone far from the mixing layer in the fast flow zone, it is assumed to be representative also in the mixing layer and further within the slow flow zone. This may be attributed to the fact that the sum of resistance forces in the slow flow zone remains approximately constant regardless of the increase in the drag. The kinematic eddy viscosity may be correlated to the total resistance force. ${ }^{17)}$
Equations (1)-(2) are rendered dimensionless with the use of the following expressions:

$(\widetilde{U}, \tilde{V})=\widetilde{U}_{f}(U, V), \quad \widetilde{H}=\widetilde{H}_{f} H$, $\tilde{x}=\tilde{B}_{f} x, \quad \tilde{y}=\frac{\tilde{B}_{f}}{\delta} y, \quad \tilde{t}=\frac{\tilde{B}_{f}}{\widetilde{U}_{\infty}} t$.

$(4 a, b, c, d, e)$

The parameter $\delta$ is introduced to adjust the normalised mixing layer width $(1-\phi) /\left.\frac{d U_{0}}{d y}\right|_{y=0}$ to the value of 2 in all cases described in this paper, where $\phi=\widetilde{U}_{s} / \widetilde{U}_{f}$ is the ratio of undisturbed velocities located far from the shear layer and $U_{0}$ is the base flow velocity. Introducing the above expressions into (1)-(2) yields

$$
\begin{gathered}
\frac{\partial U}{\partial t}+U \frac{\partial U}{\partial x}+\delta V \frac{\partial U}{\partial y}=-\frac{1}{F^{2}} \frac{\partial H}{\partial x}+\beta-\beta \frac{T_{b x}}{H}+\frac{1}{R} \frac{\partial^{2} U}{\partial x^{2}}+\frac{\delta^{2}}{R} \frac{\partial^{2} U}{\partial y^{2},} \\
\frac{\partial V}{\partial t}+U \frac{\partial V}{\partial x}+\delta V \frac{\partial V}{\partial y}=-\frac{\delta}{F^{2}} \frac{\partial H}{\partial y}-\beta \frac{T_{b y}}{H}+\frac{1}{R} \frac{\partial^{2} V}{\partial x^{2}}+\frac{\delta^{2}}{R} \frac{\partial^{2} V}{\partial y^{2}}, \\
\frac{\partial H}{\partial t}+\frac{\partial U H}{\partial x}+\delta \frac{\partial V H}{\partial y}=0,
\end{gathered}
$$

where the bed friction parameter $\beta$, the Froude number $F$ and the kinematic eddy viscosity based Reynolds number $R$ are defined as

$$
\beta=\frac{C_{f} \widetilde{B}_{f}}{\widetilde{H}_{f}}, F=\frac{\widetilde{U}_{f}}{\left(\tilde{g} \widetilde{H}_{f}\right)^{1 / 2}}, R=\frac{\widetilde{U}_{f} \widetilde{B}_{f}}{\widetilde{v}_{T}} .
$$

It is assumed that the channel is sufficiently wide to allocate a shear layer which does not reach the side walls. As a consequence, the transverse velocity $V$ vanishes and the streamwise velocity $U$ asymptotically approaches to constant slip velocities at the side walls:

$$
\frac{\partial U}{\partial y}=0 \text { at } y=-B_{S}, B_{f}, \quad V=0 \text { at } y=-B_{S}, B_{f},
$$

where the normalized widths of the slow and fast flow zones, $B_{s}=\tilde{B}_{s} / \tilde{B}_{f} \delta$ and $B_{f}=\delta$, are calculated from equation (4d). A sketch of the normalized flow velocity distribution along the channel cross-section is depicted in Fig. 1.

\section{(1) Asymptotic expansions}

Perturbation expansions are introduced to the velocity components and the flow depth in the form:

$$
\begin{gathered}
U(x, y, t)=U_{0}(y)+A U_{1}(y) e^{i(k x-\omega t)}, \\
V(x, y, t)=A V_{1}(y) e^{i(k x-\omega t)}, \\
H(x, y, t)=1+A H_{1}(y) e^{i(k x-\omega t)},
\end{gathered}
$$

where $A$ is the amplitude, assumed to be small in the linear reach, such that $A^{2}$ vanishes, $\omega$ is the angular frequency and $k=k_{r}+\mathrm{i} k_{i}$, where $k_{r}$ is

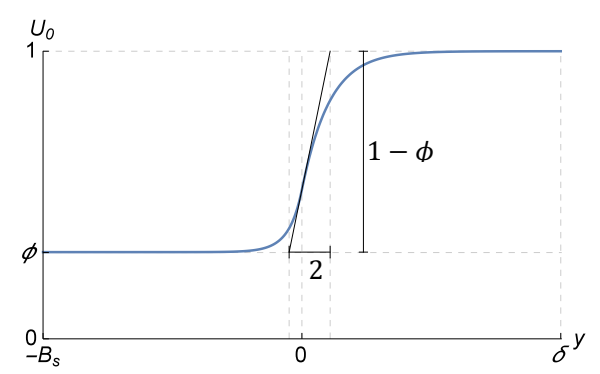

Fig. 1 Base flow velocity distribution along the channel crosssection. Normalized variables are shown. 
the wavenumber and $k_{i}$ is the growth rate of perturbation. In the scheme of the present analysis, we consider downstream growing perturbations, for which corresponds $k_{i}<0$.

\section{LINEAR STABILITY ANALYSIS}

\section{(1) Case 1}

First, based on Michalke, ${ }^{10,11)}$ we assume an inviscid shear layer without bottom shear stress. The governing equations (4)-(6) are then reduced to

$$
\begin{gathered}
\frac{\partial U}{\partial t}+U \frac{\partial U}{\partial x}+\delta V \frac{\partial U}{\partial y}=-\frac{1}{F^{2}} \frac{\partial H}{\partial x} \\
\frac{\partial V}{\partial t}+U \frac{\partial V}{\partial x}+\delta V \frac{\partial V}{\partial y}=-\frac{\delta}{F^{2}} \frac{\partial H}{\partial y}, \\
\frac{\partial H}{\partial t}+\frac{\partial U H}{\partial x}+\delta \frac{\partial V H}{\partial y}=0 .
\end{gathered}
$$

We then assume that the base flow velocity $U_{0}(y)$ is given by the following hyperbolic tangent profile modified from Michalke: ${ }^{10,11)}$

$$
U_{0}(y)=\phi+0.5(1-\phi)(1+\tanh y) .
$$

\section{(2) Case 2}

In case 2, based on Chu, ${ }^{13)}$ we assume an inviscid shear layer with bottom shear stress. In addition, we assume that the velocity gradient is caused by an extra drag in the slow flow zone. The governing equations (4)-(6) are then reduced to

$$
\begin{gathered}
\frac{\partial U}{\partial t}+U \frac{\partial U}{\partial x}+\delta V \frac{\partial U}{\partial y}=-\frac{1}{F^{2}} \frac{\partial H}{\partial x}+\beta\left(1-\frac{T_{b x}}{H}\right), \\
\frac{\partial V}{\partial t}+U \frac{\partial V}{\partial x}+\delta V \frac{\partial V}{\partial y}=-\frac{\delta}{F^{2}} \frac{\partial H}{\partial y}-\beta \frac{T_{b y}}{H}, \\
\frac{\partial H}{\partial t}+\frac{\partial U H}{\partial x}+\delta \frac{\partial V H}{\partial y}=0,
\end{gathered}
$$

Given that the velocity gradient is generated by a differential drag, the normalized bed shear stress vector is expressed as

$$
\left(T_{b x}, T_{b y}\right)=\left(1+\frac{C_{f s}}{C_{f}}\right)\left(U^{2}+V^{2}\right)^{1 / 2}(U, V),
$$

where $C_{f s}=C_{f}\left(1-\phi^{2}\right) / \phi^{2}$ is the extra drag coefficient in the slow flow zone. The term containing $C_{f s}$ is dropped in the fast flow zone. We assume that equation (16) holds in case 2. We also assume that right at the boundary between the slow and fast flow zones, the velocities, the flow depth and the shear stresses are continuous, yielding

$$
\begin{aligned}
& \lim _{\mathrm{y} \rightarrow+0}\left(U, V, H, \frac{\partial U}{\partial x}, \frac{\partial U}{\partial y}+\frac{\partial V}{\partial x}, \frac{\partial V}{\partial y}\right) \\
&=\lim _{\mathrm{y} \rightarrow-0}\left(U, V, H, \frac{\partial U}{\partial x}, \frac{\partial U}{\partial y}+\frac{\partial V}{\partial x}, \frac{\partial V}{\partial y}\right) .
\end{aligned}
$$

\section{(3) Case 3}

In case 3, we retain all terms in equations (4)-(6). Considering the base flow, only, equations (4)-(6) take the form

$\beta\left(1-U_{0}^{2}\right)+\epsilon \frac{d^{2} U_{0}}{d y^{2}}=0$, for the fast flow zone,
$\beta\left[1-\left(\frac{U_{0}}{\phi}\right)^{2}\right]+\epsilon \frac{d^{2} U_{0}}{d y^{2}}=0$, for the slow flow zone,

The analytical solution of the above equations under conditions (8) and (20) yields, respectively:

$$
\begin{gathered}
U_{0}=3 \tanh ^{2}\left[\tanh ^{-1}\left(\frac{\psi+2}{3}\right)^{1 / 2}+\left(\frac{\beta R}{2}\right)^{1 / 2} y\right]-2, \\
U_{0}=3 \phi \operatorname{coth}^{2}\left[\operatorname{coth}^{-1}\left(\frac{\psi+2 \phi}{3 \phi}\right)^{1 / 2}-\left(\frac{\beta R}{2 \phi}\right)^{1 / 2} y\right]-2 \phi,
\end{gathered}
$$

where $\psi$ is streamwise velocity at the interface between the two zones,

$$
\psi=\left(\frac{2 \phi^{2}}{1+\phi}\right)^{1 / 3} .
$$

\section{(4) Solutions at $O(A)$}

Herein, we give details of the solutions at $O(A)$ for case 3 . For cases 1 and 2 , a similar procedure is taken, yielding simpler equations.

The equations at $O(A)$ in case 3 take the following form in the fast flow zone:

$$
\begin{gathered}
{\left[i\left(k U_{0}-\omega\right)+\frac{k^{2}}{R}+2 \beta U_{0}-\frac{1}{R} \frac{d^{2}}{d y^{2}}\right] U_{1}+\frac{d U_{0}}{d y} V_{1}} \\
+\left(i k F^{-2}-\beta U_{0}^{2}\right) H_{1}=0, \\
{\left[i\left(k U_{0}-\omega\right)+\frac{k^{2}}{R}+\beta U_{0}-\frac{1}{R} \frac{d^{2}}{d y^{2}}\right] V_{1}+F^{-2} \frac{d H_{1}}{d y}=0,} \\
i k U_{1}+\frac{d V_{1}}{d y}-i\left(\omega-k U_{0}\right) H_{1}=0,
\end{gathered}
$$

and the following form in the slow flow zone:

$$
\begin{gathered}
{\left[i\left(k U_{0}-\omega\right)+\frac{k^{2}}{R}+2 \frac{\beta}{\phi} U_{0}-\frac{1}{R} \frac{d^{2}}{d y^{2}}\right] U_{1}+\frac{d U_{0}}{d y} V_{1}} \\
+\left(i k F^{-2}-\beta U_{0}^{2}\right) H_{1}=0 \\
{\left[i\left(k U_{0}-\omega\right)+\frac{k^{2}}{R}+\frac{\beta}{\phi} U_{0}-\frac{1}{R} \frac{d^{2}}{d y^{2}}\right] V_{1}+F^{-2} \frac{d H_{1}}{d y}=0} \\
i k U_{1}+\frac{d V_{1}}{d y}-i\left(\omega-k U_{0}\right) H_{1}=0
\end{gathered}
$$

In order to solve (27)-(32) with its corresponding boundary conditions, $U_{1}, V_{1}$, and $H_{1}$ are expanded employing the Chebyshev polynomials in the following form in the fast flow zone:

$$
\begin{gathered}
U_{1}=\sum_{j=0}^{N} a_{j} T_{j}(\xi), V_{1}=\sum_{j=0}^{N} a_{(N+1)+j} T_{j}(\xi), \\
H_{1}=\sum_{j=0}^{N} a_{2(N+1)+j} T_{j}(\xi),
\end{gathered}
$$

and in the following form in the slow flow zone:

$$
\begin{gathered}
U_{1}=\sum_{j=0}^{N} a_{3(N+1)+j} T_{j}(\gamma), V_{1}=\sum_{j=0}^{N} a_{4(N+1)+j} T_{j}(\gamma), \\
H_{1}=\sum_{j=0}^{N} a_{5(N+1)+j} T_{j}(\gamma),
\end{gathered}
$$

where $a_{j}(j=0,1, \ldots, 6 N+5)$ are the coefficients of the Chebyshev polynomials and $T_{j}(\xi)$ and $T_{j}(\gamma)$ are the Chebyshev polynomials in $\xi$ and $\gamma$ of degree $j$ respectively. The independent variables $\xi$ and $\gamma$ both range from -1 to 1 , and are related 
to $y$ by the equations $\xi=2 y / \delta-1(0 \leq y \leq \delta)$ and $\gamma=2 y / B_{s}+1\left(-B_{s} \leq y \leq 0\right)$, respectively. The expansions (33)-(34) are substituted into the systems (27)-(29) and (30)-(32), respectively. The resulting six equations are evaluated at the Gauss-Lobatto points defined by

$$
\xi_{m}=\cos \frac{m \pi}{N}, \quad \lambda_{m}=\cos \frac{m \pi}{N},
$$

where $m=0,1,2, \ldots, N$. Therefore, the number of points where the governing equations are evaluated is $N+1$. A system of $6(N+1)$ algebraic equations with $6(N+1)$ unknown coefficients $a_{0}, a_{1}, a_{2}, \ldots, a_{6 N+5}$ is obtained. Eight equations of the system are then replaced by the four boundary conditions (9) and four of the matching conditions (21). The resulting algebraic system takes the form

$$
\mathcal{M} \boldsymbol{A}=0 \text {, }
$$

where $\mathcal{M}$ is a $6(N+1) \times 6(N+1)$ matrix in which the elements are derived from the coefficients of $U_{1}, V_{1}$ and $H_{1}$ in the expanded forms of the governing equations and boundary and matching conditions, and $\boldsymbol{A}=\left[a_{0}, a_{1}, \ldots, a_{6 N+5}\right]^{T}$. The condition for (36) to have a non-trivial solution is that $\mathcal{M}$ should be singular. Thus,

$$
|\mathcal{M}|=0 \text {. }
$$

In the scope of spatial analysis, it is convenient to decompose the matrix $\mathcal{M}$ in the form

$$
\mathcal{M}=\mathcal{L}+k \mathcal{R}_{1}+k^{2} \mathcal{R}_{2},
$$

where the entries of $\mathcal{L}$ are composed by the terms of the entries of $\mathcal{M}$ which do not contain $k$ (or $k^{2}$ ), and $\mathcal{R}_{1}$ and $\mathcal{R}_{2}$ are composed by the coefficients of $k$ and $k^{2}$, respectively, in the entries of $\mathcal{M}$. Substituting the above decomposition into (37) yields the following nonlinear eigenproblem:

$$
\mathcal{L} \boldsymbol{A}=-\left(k \mathcal{R}_{1}+k^{2} \mathcal{R}_{2}\right) \boldsymbol{A} \text {. }
$$

The above expression is linearized in the form

$$
\left(\begin{array}{cc}
\mathcal{L} & 0 \\
0 & I
\end{array}\right)\left(\begin{array}{c}
\boldsymbol{A} \\
k \boldsymbol{A}
\end{array}\right)=k\left(\begin{array}{cc}
-\mathcal{R}_{1} & -\mathcal{R}_{2} \\
I & 0
\end{array}\right)\left(\begin{array}{c}
\boldsymbol{A} \\
k \boldsymbol{A}
\end{array}\right),
$$

where $I$ is the identity matrix. The solution of the above equation takes the functional form

$$
k=k(\omega, \beta, R, \phi, F) .
$$

For cases 1 and 2 , the eigenvalue problem expressed in (38) takes the linear form

$$
\mathcal{M}=\mathcal{L}+k \mathcal{R}_{1},
$$

with $\mathcal{M}, \mathcal{L}$ and $\mathcal{R}_{1}$ being distinct in each case.
In the results presented in the following section, we assume that the widths of the fast and slow flow re the same, therefore $B_{v}=\delta$. In addition, unless otherwise mentioned, we employ $F=0.5$.

\section{RESULTS}

\section{(1) Growth rate of perturbations}

Following Gaster, ${ }^{19)}$ the spatial growth rate $k_{i}$ can be alternatively determined from the temporal analysis as being

$$
k_{i}=-\omega_{i} / c_{g},
$$

where $c_{g}=\partial \omega_{r} / \partial k$ is the group velocity. In the temporal analysis, the wavenumber is real, while the angular frequency is complex as $\omega=\omega_{r}+\mathrm{i} \omega_{i}$, where $\omega_{r}$ is the real angular frequency and $\omega_{i}$ is the temporal growth of perturbations.

Fig. 2 depicts instability diagrams of spatial growth rates determined from the temporal and the spatial analyses. The curves of the temporal and spatial analyses are roughly similar, with a small discrepancy observed mainly in the range of large values of the growth rates. This discrepancy is expected because equation (43) is obtained assuming that the terms of order $O\left(\max \left(\omega_{i}^{2}\right)\right)$ are negligible. ${ }^{19)}$ The discrepancy is more significant for cases where $\phi$ and $\beta$ are small and $R$ is large.

Kimura et al. ${ }^{20)}$ employed momentum equations for an open channel flow adjacent to a dead flow zone which contained source terms accounting for the effects of bed shear stress and Reynolds stress. However, these source terms were dropped for simplicity in their linear stability analysis. In the present work, for $\phi=0.3$, the inclusion of the bed shear stress effect resulted in reduction of the unstable domains, verified by comparing Fig. 2(a) and (b). This is because the effect of the bottom friction inhibits the effect of the lateral velocity gradient. ${ }^{9,13,15)}$ The inclusion of the Reynolds stress effect changes remarkably the base flow velocity profile. When $R$ is large, the velocity gradient is abrupt, resulting in a large unstable domain, whereas the decrease of $R$ mildens the velocity gradient, resulting in a smaller unstable domain.

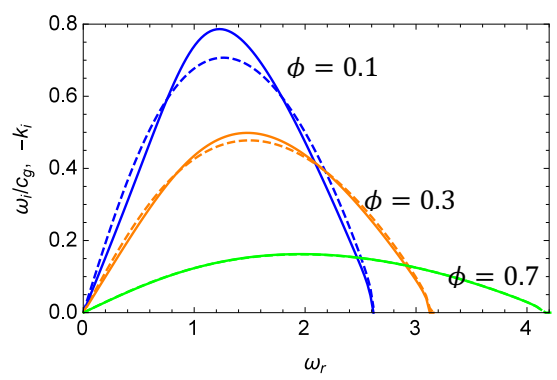

a) Case 1

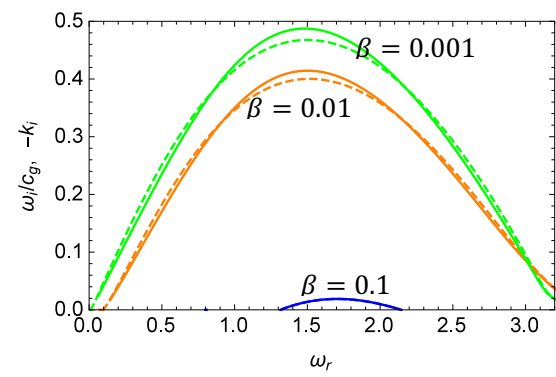

b) Case 2, $\phi=0.30$

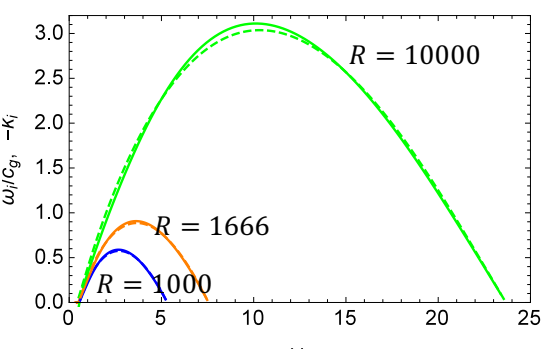

c) Case 3, $\phi=0.30, \beta=0.05$

Fig. 2 Spatial growth rates obtained from the temporal analysis (dashed lines) and the spatial analysis (continuous lines). 


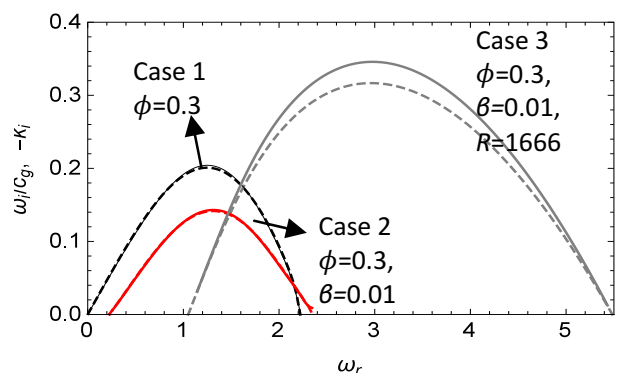

Fig. 3 Spatial growth rates obtained from the temporal (dashed lines) and spatial analysis (continuous lines), for $F=2$.

Fig 3 depicts instability diagrams of spatial growth rates determined from the temporal and the spatial analyses for a larger Froude number $F=2$. The unstable domains are reduced compared to those depicted in Fig. 2 because rapid flow plays stabilizing effects for lateral velocity gradients. ${ }^{\text {(5) }}$

\section{(2) Phase velocity}

Fig. 4 depicts curves of the phase velocity $c_{p}=\omega_{r} / k_{r}$ as a function of the frequency $\omega_{r}$, for the temporal and spatial approaches, and for multiple values of $\phi$ (case 1), $\beta$ (case 2) and $R$ (case 3 ). The ratio of undisturbed velocities $\phi$ has a strong effect on $c_{p}$, as depicted in Fig. 4(a). In Case 1 , the phase velocity in the temporal approach is constant, similar to Michalke, ${ }^{10,11)}$ and equal to $U_{0}$ at $y=0$, whereas the phase velocity varies strongly in the spatial case with the frequency $\omega_{r}$, especially for small values of $\omega_{r}$. Michalke ${ }^{11)}$ stated that the behavior of the phase velocity in the spatial predictions was observed in the experiments of Freymuth. ${ }^{16)}$

In case 2 , and more explicitly in case 3 , the phase velocity in the temporal problem is not constant, as seen in Fig. 4(b)-(c). Although a discrepancy can be noted between the temporal and spatial curves of $c_{p}$ in cases 2 and 3, mainly in the range of frequencies smaller than that where the spatial and temporal curves intersect, this discrepancy is in general less significant than that verified in case 1 .

The curves in the temporal and spatial cases intersect each other near the points where $\omega_{i}$ and $-k_{i}$ are maximized. Therefore, the preferential frequencies for maximum amplification in time and space converge to approximately the same value. This means that both temporal and spatial approaches can lead to reliable results for the preferential growth rate in the onset of instability development.

\section{(3) Eigenfunctions}

In their evaluation of eigenfunctions, Michalke $^{10,11)}$ and Freymuth ${ }^{21)}$ demonstrated that the distribution of the disturbance velocity component across the spanwise domain can be captured by the spatial analysis alone. In particular, the temporal analysis carried out by Michalke ${ }^{10)}$ resulted in symmetric and anti-symmetric cross-distributions of the real and imaginary parts the eigenfunction, respectively. This behavior was not observed in Michalke's ${ }^{11}$ spatial analysis, where the cross-distribution of the real and imaginary parts of the eigenfunctions were asymmetric. Moreover, a sharp phase reversal of the disturbance velocity component, observed in the experiments of Freymuth, ${ }^{16)}$ was predicted by the spatial analysis by Michalke, ${ }^{11)}$ but not in the temporal analysis, ${ }^{10)}$ except in the case of a neutral disturbance.

Fig. 5 depicts results for the real and imaginary parts of the eigenfunction $U_{1}(y)$ across the spanwise domain. The eigenfunction is normalized such that $\operatorname{Re}\left(U_{1}(0)\right)=1$. The presented curves are obtained for the wavenumber and frequency where the amplification is maximized. The cross-distributions of the eigenfunction $U_{1}(y)$ from both the temporal and spatial analyses are found to be asymmetric for case 3, while in the temporal approach of case 1, the cross-distribution of the real part is symmetric and the cross-distribution of the imaginary part is anti-symmetric, similar to Michalke. ${ }^{10)}$ Moreover, while in the temporal distributions of Fig. 5(a) and (b) the locations of the inflections of $\operatorname{Re}\left(U_{1}\right)$ and $\operatorname{Im}\left(U_{1}\right)$, respectively, are distinct $(y \approx-1.5$ and $y \approx-2.0$, respectively), for the temporal distributions of case 1 and both the temporal and spatial distributions of case 3 , the locations are virtually coincident, indicating sharp phase reversals.

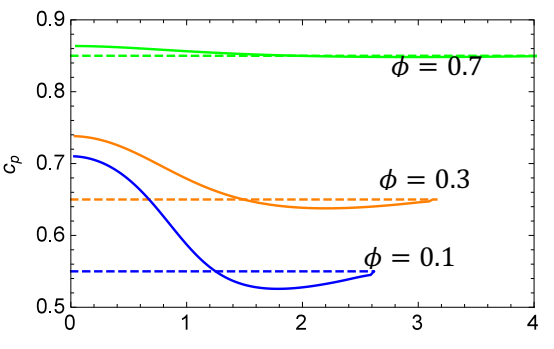

a) Case 1

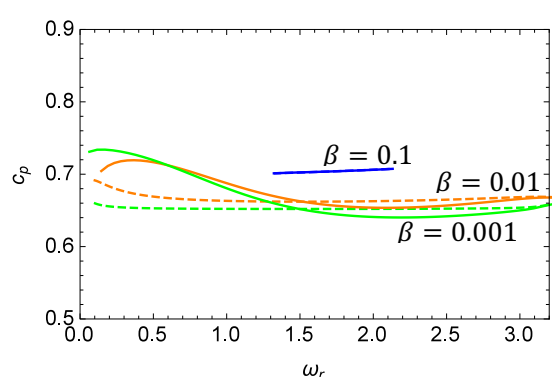

b) Case 2, $\phi=0.30$

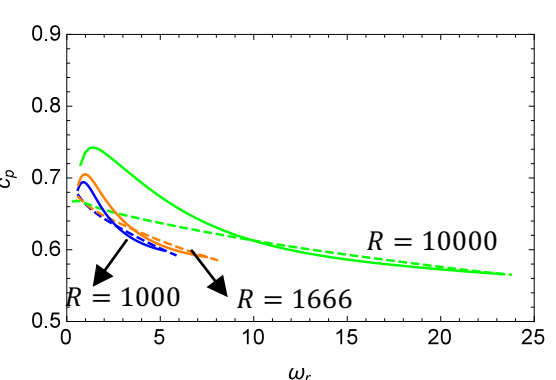

c) Case $3, \phi=0.30, \beta=0.05$

Fig. 4 Phase velocities obtained from the temporal analysis (dashed lines) and the spatial analysis (continuous lines). 


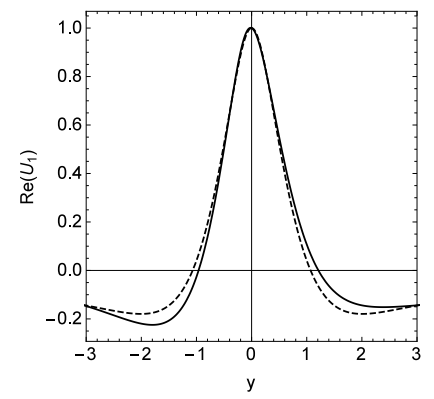

a) Case 1

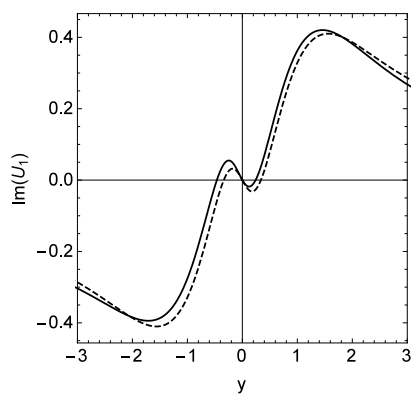

b) Case 1

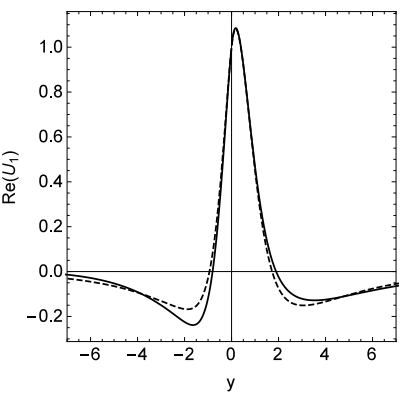

c) Case 3

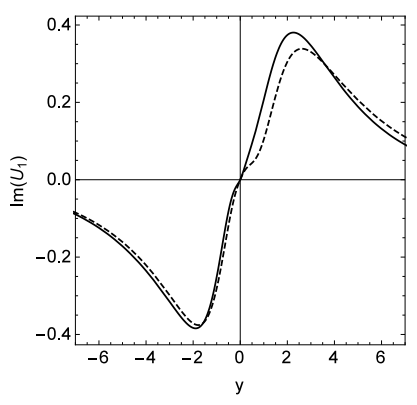

d) Case 3

Fig. 5 Lateral distribution of the real and imaginary parts of eigenfunction $U_{1}(y)$ at the maximum amplification in time (dashed curves) and space (continuous curves), for cases 1 and 3 .

\section{CONCLUSIONS}

In this work, less difference was found between the results from the temporally and spatially growing disturbances in viscous approaches than those from inviscid approaches. In the viscous approach, the phase velocity was determined to be correlated to the frequency not only in the spatial case, but also in the temporal case. Additionally, the cross-distribution of the streamwise velocity eigenfunction was found to be asymmetric for both the temporal and spatial cases in the viscous approach with bed shear.

The most noticeable difference between results from the temporal and spatial analyses for viscous flow with bed shear may be attributed to the phase velocity in the range of small frequencies. This is more clearly observed in the case of large eddy viscosity based Reynolds number $R$ and resembles the discrepancy between results from spatial and temporal linear stability analysis in inviscid shear layers.

\section{REFERENCES}

1) Fischer, H. B., List, E. J., Koh, R. C. J., Imberger, J. and Brooks, N. H.: Mixing in inland and coastal waters. Academic, New York, 1979.

2) Ikeda, S., Izumi, N. and Itoh, R.: Effects of pile dikes on flow retardation and sediment transport. J. Hydraul. Eng., Vol.117, pp.1459-1478, 1991.

3) Tsujimoto, T. and Kitamura, T.: Lateral bed-load transport and sand-ridge formation near vegetation zone in an open channel. J. Hydrosci. Hydraul. Eng., Vol.13, pp.35-45, 1995.

4) Prooijen, B. C. and Uijttewaal, W. S. J.: On the initiation of large scale turbulence structures in the numerical simulation of shallow mixing layers. River Flow 2002 - Proceedings of the International Conference on Fluvial Hydraulics, 2002.

5) Uijttewaal, W. S. J. and Booij, R.: Effects of shallowness on the development of free-surface mixing layers. Phys. Fluids, Vol. 12, pp.392-402, 2000.

6) Kolyshkin, A. A., and Ghidaoui, M. S.: Gravitational and shear instabilities in compound and composite channels. $J$. Hydraul. Eng., Vol.128, pp.1076-1086, 2002.

7) Ikeda, S., Ohta, K., and Hasegawa, H.: Instability-induced horizontal vortices in shallow open-channel flows with an inflection point in skewed velocity profile. J. Hydrosci. Hydraul. Eng., Vol.12, pp.69-84, 1994.

8) Prooijen, B. C. and Uijttewaal, W. S. J.:A linear approach for the evolution of coherent structures in shallow mixing layers. Phys. Fluids, Vol.14, pp.4105-4114, 2002.

9) White, B. L. and Nepf, H. M.: Shear instability and coherent structures in shallow flow adjacent to a porous layer. $J$. Fluid Mech., Vol.593, pp.1-32, 2007.

10) Michalke, A.: On the inviscid instability of hyperbolic tangent velocity profile. J. Fluid Mech., Vol.19, pp.543-556, 1964.

11) Michalke, A.: On spatially growing disturbances in an inviscid shear layer. J. Fluid Mech., Vol.23, pp.521-544, 1965.

12) Tamai, N., Asaeda, T., and Ikeda, H.: Study on generation of periodical large surface eddies in a composite channel flow. Water Resour. Res., Vol.22, pp.1129-1138, 1986.

13) Chu, V. H., Wu, J.-H., and Khayat, R. E.: Stability of transverse shear flows in shallow open channels. J. Hydraul. Eng., Vol.117, pp.1370-1388, 1991.

14) Ghidaoui, M. S., and Kolyshkin, A. A.: Linear stability analysis of lateral motions in compound open channel. $J$. Hydraul. Eng., Vol.125, pp.871-880, 1999.

15) Lima, A. C. and Izumi, N.: Linear stability analysis of open-channel shear flow generated by vegetation. J. Hydraul. Eng., Vol.140, pp.231-240, 2014.

16) Freymuth, P.: Diss. Technische Universität Berlin (as cited in Michalke, 1965), 1965.

17) Lima, A. C. and Izumi. N.: On the nonlinear development of shear layers in partially vegetated channels. Phys. Fluids, Vol.26, pp. 084109-1-084109-22, 2014

18) Tsujimoto, T.: Open channel flow with bank vegetation. KHL Communication, 2, Dept. of Civil Engineering, Kanazawa University., pp.41-54, 1991.

19) Gaster, M.: A note on the relation between the temporally-increasing and spatially-increasing disturbances in hydrodynamic instability. J. Fluid Mech., Vol.14, pp.222-224, 1962.

20) Kimura, I., Hosoda, T., and Tomochika, H. Characteristics of spatially growing disturbances in a mixing shear layer of open channel flows. J. Hydraul. Coastal and Environmental Engineering, JSCE, No.509/II-30, pp. 99-109, 1995.

21) Freymuth, P. On transition in a separated laminar boundary layer. J. Fluid Mech., Vol.25, pp.683-704, 1966.

(Received September 30, 2015) 